\title{
NECESSIDADE DE REMANEJAMENTO ENTRE SETORES: PERCEPÇÃO DOS TÉCNICOS DE ENFERMAGEM EM UM HOSPITAL
}

\section{THE NECESSITY OF WORK REASSIGNMENT BETWEEN SECTORS: PERCEPTION OF NURSING TECHNICIANS IN A HOSPITAL}

\section{NECESIDAD DE REUBICACIÓN ENTRE SECTORES: LA PERCEPCIÓN DE LOS TÉCNICOS EN ENFERMERÍA EN UN HOSPITAL}

Humberto Ferreira de Oliveira Quites ${ }^{1}$, Cristiane de Oliveira Moreira ${ }^{2}$, Fabiana do Carmo Vieira ${ }^{2}$, Giorgia Emilly Ferreira Carvalho Corso $^{3}$, Marina Celly Martins Ribeiro de Souza ${ }^{4}$, Edna Lúcia Campos Wingester ${ }^{5}$

\section{RESUMO}

Objetivo: Analisar as percepções dos técnicos de enfermagem de um hospital de pequeno porte sobre o remanejamento entre setores. Método: Pesquisa descritiva, com abordagem qualitativa, em que os dados foram coletados por meio de entrevistas com trinta técnicos de enfermagem, utilizando-se o método de saturação. 0 material foi analisado a partir da análise de conteúdo proposta por Bardin. Resultados: Os resultados apontaram que o remanejamento é considerado, pelos colaboradores, uma situação eventual e necessária à rotina de trabalho. Entretanto, pode-se observar que causa desconforto à equipe e que deve ser realizado com critérios que não impactem no processo de trabalho dos envolvidos. Os pontos negativos elencados pelos colaboradores, neste processo, foram a sobrecarga de trabalho e a mudança de setor, entretanto os mesmos citaram a possibilidade de aprendizado e aperfeiçoamento profissional, como pontos positivos do remanejamento. Conclusão: 0 remanejamento é percebido como parte da rotina pelos profissionais, sendo necessário um investimento, por parte da gestão, para prevenir situações de desconforto e desarranjos que impactem na saúde ocupacional de seus colaboradores bem como na qualidade da assistência de seus pacientes.

Descritores: Enfermagem; Gestão em saúde; Prática profissional; Administração de recursos humanos em hospitais.

\begin{abstract}
Objective: To analyze the perceptions of nursing technicians in a small hospital in Belo Horizonte about work reassignment between sectors. Method: This was an exploratory study with a qualitative approach in which the data were collected through interviews with thirty nursing technicians by applying the saturation method. The data were analyzed through the content analysis proposed by Bardin. Results: The results indicated that work reassignment is considered by employees as an eventual and necessary event in their work routine. However, it is noticed that it causes discomfort to team members and must be carried out with criteria in order to not impact the work process of those involved. The negative aspects mentioned in this process were work overload and sector change. However, the participants mentioned the possibility of learning and professional development as positive aspects. Conclusion: Work reassignment is perceived by professionals as part of the work routine, requiring an investment by the management to prevent discomfort and disarray situations that impact the occupational health of employees and patients' quality care.
\end{abstract}

Descriptors: Nursing; Health management; Professional practice; Personnel administration, Hospital.

\section{RESUMEN}

Objetivo: Analizar las percepciones de los técnicos de enfermería de un hospital pequeño acerca de la reubicación de sectores. Método: Se trata de una investigación exploratoria, con enfoque cualitativo, en el que se recogieron los datos a través de entrevistas con treinta (30) técnicos de enfermería, y se utiliza el método de saturación. Todo el material fue analizado a partir del análisis de contenido propuesto por Bardin. Resultados: Nuestros resultados mostraron que la reubicación es considerada por los empleados como sea posible y necesario en la situación de su rutina de trabajo. Sim embargo, se puede observar que esto causa molestias al equipo y debe llevarse a cabo con criterios que no impactan en el proceso de trabajo de los involucrados. Hay aspectos negativos de este proceso como la carga de trabajo y el cambio de sector. Sin embargo, los participantes se refirieron a la posibilidad de aprendizaje y desarrollo profesional. Conclusión: La reubicación se percibe como parte de la rutina profesional, que requiere una inversión de la dirección para evitar situaciones de malestar y trastornos que afectan a la salud en el trabajo de sus empleados y la calidad de atención a sus pacientes.

Descriptores: Enfermería; Gestión em salud; Práctica profesional; Administración de personal en hospitales.

${ }^{1}$ Graduado em Enfermagem. Doutorado em Enfermagem pela Universidade Federal de Minas Gerais. Professor Adjunto A na Universidade Federal de São João del-Rei. ${ }^{2}$ Graduada em Enfermagem pelo Centro Universitário Belo Horizonte. ${ }^{3}$ Graduada em Enfermagem pela Universidade de Itaúna. ${ }^{4}$ Graduada em Enfermagem. Doutora em Enfermagem pela Universidade Federal de Minas Gerais. Docente em The College of New Jersey - Department of Public Health. ${ }^{5}$ Graduada em Enfermagem. Doutora em Enfermagem pela Universidade Federal de Minas Gerais. Docente no Centro Universitário Newton Paiva.

Como citar este artigo:

Quites HFO, Moreira CO, Vieira FC, et al. Necessidade de remanejamento entre setores: percepção dos técnicos de enfermagem em um hospital. 2017;7:e1799. [Access__]; Available in:___. https://doi.org/10.19175/recom.v7i0.1799 


\section{INTRODUÇÃO}

A institucionalização da enfermagem como profissão, a partir de meados do século XIX, proporcionou uma reorganização dos processos de trabalho. No Brasil, a divisão técnica da enfermagem deu origem a diferentes categorias como o técnico, o auxiliar em enfermagem e as parteiras. Neste contexto, maior autonomia é garantida ao profissional enfermeiro, responsável pelo desempenho privativo de atividades relacionadas ao ensino, supervisão dos serviços de enfermagem, assistência ao paciente crítico e administração das rotinas de trabalho ${ }^{(1)}$.

0 enfermeiro assume atividades complexas, sendo necessário lidar, muitas vezes, com situações e/ou eventos adversos, nos quais suas decisões e responsabilidades devem ser compartilhadas com a equipe em prol da instituição $0^{(2)}$.

Várias são as atividades essenciais para o bom desempenho do cuidado em ambiente hospitalar, tais como: elaboração de escala, remanejamento de funcionários, dentre outros serviços considerados administrativos ${ }^{(3)}$. Problemas e situações conflituosas, como a ausência de um ou mais membros da equipe durante a jornada de trabalho e os afastamentos por motivo de doenças, acontecem neste contexto e podem refletir na qualidade da assistência de enfermagem, bem como em todo o processo de trabalho ${ }^{(4)}$.

Quando o absenteísmo supera o planejamento, faz-se necessário o remanejamento de profissionais entre os setores do serviço hospitalar, podendo impactar negativamente na satisfação dos colaboradores envolvidos ${ }^{(5)}$. Além disso, a ausência de um ou mais membros da equipe de enfermagem pode contribuir para a sobrecarga de trabalho dos demais colaboradores, aumentando o desgaste físico, psicológico, social e espiritual destes e, consequentemente, elevando o risco de acidentes de trabalho ${ }^{(5)}$.

Para garantir uma assistência eficiente é necessário refletir sobre a saúde e qualidade de vida dos técnicos em enfermagem que estão diretamente ligados à prestação de cuidado ao paciente no âmbito hospitalar, considerando-se as horas trabalhadas, qualidade do ambiente, as tecnologias empregadas, entre outros ${ }^{(6)}$. Deste modo, deve-se sempre proporcionar espaços e decisões que garantam a satisfação do profissional, mesmo com as ausências não programadas e a necessidade de remanejamento.

A insatisfação dos profissionais com o processo de remanejamento de setores e horários de trabalho no ambiente hospitalar não é uma dificuldade enfrentada apenas no Brasil. Em países como a Alemanha e Finlândia ${ }^{(7-8)}$, estudos têm apontado a geração de situações conflituosas e insatisfatórias diante desta temática, sobretudo por não haver agenda de trabalho fixo, o que contribui, de sobremaneira, para o desgaste dos membros da equipe de enfermagem.

Neste contexto, percebe-se que ainda é necessário conhecer mais sobre os aspectos relacionados ao remanejamento de profissionais técnicos em enfermagem a partir do ponto de vista destes sujeitos. Acredita-se que este conhecimento permitirá o desenvolvimento de estratégias que minimizem os possíveis impactos da mudança, trazendo benefícios tanto para os colaboradores quanto para as instituições, para manutenção de uma assistência de qualidade ao cliente institucionalizado.

Diante do exposto, questiona-se: quais as percepções dos técnicos em enfermagem a respeito do remanejamento? Desta forma, este estudo tem como objetivo analisar as percepções dos técnicos em enfermagem de um hospital de pequeno porte sobre o remanejamento entre setores.

\section{MÉTODOS}

Trata-se de uma pesquisa descritiva, com abordagem qualitativa, cujo cenário foi um hospital público de pequeno porte, localizado na capital do estado de Minas Gerais, referência estadual para doenças infectocontagiosas em adultos.

Esta proposta trabalha com aspectos subjetivos, possibilitando analisar as informações coletadas pelo levantamento de dados sob diferentes perspectivas ${ }^{(9)}$. O estudo foi realizado com técnicos em enfermagem, lotados nos setores de internação dos períodos diurno e noturno. Como critério de inclusão, foi estabelecido que o participante fosse técnico em enfermagem, tivesse sido submetido ao remanejamento por mais de uma vez em toda sua atuação profissional ou nos últimos seis meses, trabalhasse nos setores de internação, e concordasse em participar da pesquisa. Os técnicos em enfermagem do centro de terapia 
intensiva não foram incluídos no estudo porque não são remanejados em função da política interna da instituição.

As entrevistas foram realizadas com trinta técnicos em enfermagem, de acordo com a disponibilidade dos participantes, dentro de um universo de 96 profissionais. As entrevistas foram encerradas tendo-se em vista o critério de saturação dos dados que, segundo Minayo ${ }^{(10)}$, deve considerar o número de sujeitos suficientes quando as falas se tornam recorrentes, porém sem desprezar informações ímpares cujo potencial explicativo tem que ser levado em conta. Os sujeitos foram numerados na ordem em que aconteciam as entrevistas, sendo nomeados como $T E$, sigla equivalente a denominação Técnico em Enfermagem. A coleta dos dados ocorreu nos meses de janeiro e fevereiro de 2016, durante os plantões dos participantes, utilizando-se um roteiro de entrevista semiestruturado e as respostas foram gravadas em mídia digital e transcritas para posterior análise. $\mathrm{O}$ uso da entrevista favoreceu a aquisição de informações contidas nos discursos dos participantes, fornecendo dados referentes a fatos, ideias, crenças, opiniões, sentimentos, condutas ou comportamentos ${ }^{(10)}$.

A análise de conteúdo das entrevistas baseou-se no referencial epistemológico proposto por Laurence $\operatorname{Bardin}^{(11)}$, caracterizada por um conjunto de instrumentos metodológicos que se aplicam a discursos extremamente diversificados. Este tipo de análise tem como referência principal um conjunto de técnicas de análises da comunicação que pode utilizar procedimentos sistemáticos e objetivos de descrição dos conteúdos apresentados pelas mensagens analisadas. Para categorização e análise dos dados, foram seguidas as três fases proposta por $\operatorname{Bardin}^{(11)}$ : (1) estabelecer as unidades de análise; (2) determinar as categorias de análise; e (3) selecionar uma amostra do material para análise.

O estudo obedeceu aos princípios éticos de pesquisas com seres humanos da resolução 466/2012 e foi aprovado por Comitê de Ética em Pesquisa Envolvendo Seres Humanos do Hospital Eduardo de Menezes/HEM/FHEMIG, segundo parecer número 1.018.207/2015.

\section{RESULTADOS E DISCUSSÃO}

A partir dos dados coletados, iniciou-se o processo de levantamento do perfil dos entrevistados. Os participantes do estudo eram predominantemente do sexo feminino (80\%) e tinham idade entre de 27 e 58 anos. Em relação a situação funcional, $83 \%$ dos técnicos de enfermagem estão no hospital há menos de 5 anos, $7 \%$ de 5 a 10 anos e $10 \%$ há mais de 10 anos.

A análise das entrevistas permitiu agrupar as falas dos participantes do estudo conforme os núcleos de sentido, dando origem a três categorias: conceito/percepção dos técnicos em enfermagem sobre o remanejamento; fatores positivos acerca do remanejamento; fatores negativos acerca do remanejamento.

\section{Conceito/percepção dos técnicos em enfermagem sobre o remanejamento}

O remanejamento de pessoal é uma prática largamente utilizada nos serviços de saúde, sobretudo nas instituições hospitalares. Mesmo em um setor onde o planejamento é evidente e a escala é bem elaborada, há possibilidade de ocorrerem ausências no quadro de profissionais, gerando a necessidade de redistribuição de seus elementos. Quando os sujeitos da pesquisa foram questionados a respeito do que é remanejamento, percebeu-se certa coerência quanto ao entendimento sobre este, o que pode estar relacionado com as vivências adquiridas pelos entrevistados ao longo da experiência profissional, bem como pelo cotidiano destes. Os participantes compreendem o remanejamento como várias ações consideradas fundamentais, do tipo: necessidade de adaptação, desfalque, equidade e reposição que, algumas vezes, geram desconforto.

TE 15: “[...] é quando um setor está desfalcado de um funcionário, aí necessita de ajuda de um outro de outro setor [...]".

TE 1: "[...] Igualar o número de funcionários de cada setor para ficar uma coisa justa".

TE 9: “[...] um setor que está precisando de funcionário no momento para $\mathrm{o}$ bem do paciente".

TE 7: “[...] para você estar se adaptando com setor diferente, rotina diferente, paciente diferente".

Neste contexto, há um entendimento sobre a possibilidade de uma eventual falta entre os membros da equipe e que o remanejamento é uma situação necessária e real no âmbito do processo de trabalho da equipe de enfermagem. Apesar das características proporcionarem diferentes sensações aos técnicos em enfermagem, a maioria compreende a 
necessidade do remanejamento, mas ressaltam a importância de existir critérios para que este seja realizado de uma forma igualitária e equilibrada. Reconhecem a importância de se definir tal redistribuição de forma organizada.

TE 5: "[...] é importante ser uma coisa equilibrada e ter critérios para realizar".

TE 14: "[...] eu não tenho problema quanto a isso, mas deveria ter um pouco de critério para que seja de forma justa".

Os profissionais da área de enfermagem representam a maior força de trabalho das instituições hospitalares e exercem, de fato, atividades que vão desde o cuidado direto ao paciente até ações gerenciais. Desta forma, torna-se importante conduzir bem este processo para minimizar possíveis impactos negativos ocasionados pela ausência de integrantes da equipe. Nesta perspectiva, o papel do enfermeiro se mostra essencial para minimizar os conflitos gerados no remanejamento ${ }^{(12)}$. O enfermeiro, responsável pela tomada de decisão, deve buscar coerência e expertise neste momento, levando em conta aspectos organizacionais, aptidões pessoais, busca pela resolutividade e qualidade, preservando a saúde ocupacional de sua equipe.

São necessárias estratégias para mitigar possíveis impactos na qualidade da assistência prestada, mantendo ou potencializando um nível adequado de satisfação dos envolvidos. A resolução 543 de 2017 do Conselho Federal de Enfermagem (COFEN) considera que, para garantir a segurança e a qualidade da assistência ao cliente, no quadro de profissionais de enfermagem, deve-se garantir sempre a continuidade ininterrupta das atividades desenvolvidas e a diversidade de atuação de sua equipe $^{(13)}$. Desta forma, mantêm-se as condições necessárias para evitar a vulnerabilidade dos envolvidos ao longo deste processo.

Um recurso utilizado no hospital estudado e evidenciado pelo grupo nas entrevistas foi a implementação de uma escala fixa de remanejamento. Esta escala foi implantada inicialmente no plantão diurno da unidade de internação com o objetivo de minimizar os conflitos gerados pela redistribuição de funcionários. Neste processo, foi acordado, junto à equipe, que um profissional, escalado previamente em cada plantão, participaria da redistribuição. Nas falas dos profissionais do plantão diurno, pode-se perceber que a definição e a deliberação do método foram bem aceitos.
TE 9: “[...] agora, com a escala fixa, melhorou bastante...".

TE 10: "[...] agora, o remanejamento ocorre de uma forma mais tranquila, que para mim teve um ganho quando a escala já vem predeterminada...".

Observa-se que houve aceitação da proposta de escala fixa, o que evita situações de desacordo ou desconforto entre os membros do plantão diurno. Entretanto, o consenso não foi geral entre os técnicos em enfermagem sobre este tipo de escala. As falas do plantão noturno evidenciam que os profissionais não aderiram à tal definição, sendo esta passível de conflitos e incongruências entre as equipes.

TE 13: "[...] eu acho que falta organização de como isso pode acontecer".

TE 17: "[...] eu acho que isso aqui é um pouco desorganizado...".

TE 23: "[...] isso aqui, no hospital, não funciona bem não. Porque sempre tem uma discussão, [...] tinha que ter um diálogo melhor e as pessoas estarem mais unidas para poderem fazer esses tipos de coisa...".

Desta forma, fica nítida a existência de divergência de opiniões entre as equipes dos diferentes turnos na decisão tomada pela gestão hospitalar. Os sujeitos do estudo identificam incipiência na comunicação sobre a deliberação do caso e se mostram insatisfeitos com a definição do remanejamento. É importante salientar que neste processo podem ocorrer entendimentos distintos sobre o processo de redistribuição de pessoal e que fatores como setores independentes e turnos podem requerer uma visão ampliada por parte do gestor.

Uma forma de evitar tais insatisfações e, por consequência, diminuir os conflitos, é o planejamento que pode ser subsidiado pelo dimensionamento do pessoal de enfermagem. Um dimensionamento bem elaborado é baseado nas características da instituição, dos profissionais de enfermagem e da clientela assistida. O dimensionamento constitui "a adequação do pessoal em termos quantitativos e qualitativos" ${ }^{\prime(14)}$ e possui como finalidade "prever a quantidade de funcionários por categoria, requerida para atender direta ou indiretamente, as necessidades de assistência de enfermagem aos pacientes" ${ }^{\prime 14)}$.

O dimensionamento é uma análise quantitativa que leva em consideração o número de pacientes do período, incluindo uma possível oscilação, e a carga de trabalho gerada pelos 
pacientes à equipe de enfermagem ${ }^{(5)}$. Quando bem aplicado, o dimensionamento do pessoal de enfermagem proporciona aos profissionais condições adequadas para a realização de suas atividades de maneira eficiente.

\section{Fatores positivos acerca do remanejamento}

Ao serem questionados sobre os pontos positivos do evento remanejamento de colaboradores, percebe-se que os participantes destacam a importância de não gerar malefício ao paciente e a necessidade de tomar a melhor decisão para que o processo de reorganização da equipe, neste tipo de situação, não impacte negativamente na qualidade da prestação de serviço.

TE 17: “[...] a necessidade do paciente não tem que sofrer nenhuma consequência devido à falta ou à ausência do funcionário[...]".

TE 8: "[...] suprir a necessidade da demanda dos pacientes do hospital".

TE 5: "Trabalho em equipe, sem sobrecarregar um bom andamento do setor, seria prestar um bom atendimento aos pacientes".

Ressalta-se que a qualidade na assistência ao paciente é entendida pelos profissionais de enfermagem como um produto que leva ao pleno atendimento das necessidades dos pacientes e dos profissionais ${ }^{(15)}$. Além deste aspecto, os colaboradores também apontam benefícios deste processo que, muitas vezes, são adquiridos nestas situações de mudança, como o crescimento profissional da equipe, ocasionada pela busca da resolutividade frente à ausência e, consequentemente, o aperfeiçoamento do trabalho em equipe que reflete na diminuição da sobrecarga de trabalho.

TE 4: "Eu acho que você cresce, você aprende, tem contato com novas clínicas e novos aparelhos [...]".

TE 10: "[...] os setores não estão ficando descobertos, e isso traz, para o funcionário que está no plantão, uma segurança [...]".

TE 16: "Seria um trabalho mais unido e tranquilo, podendo dividir melhor as tarefas com os colegas".

$\mathrm{Na}$ rotina hospitalar, a equipe de enfermagem esbarra com diferentes situações tais como: medo, relações de poder, conflitos, longas jornadas de trabalho, ansiedade, estresse, convivência com a vida e morte, tensões, entre tantas outras. Diante deste cenário, é indispensável que os gestores compreendam o trabalho da equipe de enfermagem de forma ampliada, considerando os diferentes aspectos envolvidos na prestação do cuidado, bem como a saúde física e mental de todos os seus colaboradores $^{(16)}$.

Mesmo em situações adversas, os colaboradores percebem que podem construir algo positivo, podem aprimorar, seja individual ou coletivamente. $O$ trabalho também oportuniza momentos de satisfação, uma vez que, no trabalho, o ser humano constrói a vida e se insere no mundo não somente como forma de sobreviver, mas também para realização pessoal e profissional do indivíduo ${ }^{(16)}$.

É importante que o processo de remanejamento seja consistente e bem discutido entre os envolvidos, para que as atividades sejam desenvolvidas da melhor maneira possível. Os processos de gestão que envolvem os trabalhadores são capazes de criar vínculos solidários e participativos e atendem aos anseios dos envolvidos, muitas vezes, mudando os modelos assistenciais. Espaços democráticos podem conduzir à humanização das práticas, levando a uma maior resolutividade, diminuindo os índices de problemas ocupacionais ${ }^{(17)}$.

\section{Fatores negativos acerca do remanejamento}

A sobrecarga de trabalho, ocasionada pelo remanejamento dos profissionais, devido à ausência de um ou mais colaboradores da equipe, foi compreendida pelos colaboradores como um fator que gera um desconforto no ambiente de trabalho.

TE 14: "[...] tem que olhar o critério, porque, às vezes, desafoga uma ala e aperta outra".

É importante avaliar tal carga excessiva de trabalho, uma vez que esta impacta não apenas no estado físico do colaborador, mas também no psíquico ${ }^{(18)}$. Associada a este contexto, percebese, nas falas, outros reflexos desta redistribuição como a dificuldade de adaptação em outro setor, com outra equipe e o desconforto ocasionado pelo rompimento das barreiras do desconhecido.

TE 2: "[...] de repente, você muda para outro ambiente e tem que se adaptar de novo[...]".

TE 3: "[...] esse funcionário tem que se adequar às rotinas do setor...tem que reaprender, porque os procedimentos são os mesmos, mas as rotinas são diferenciadas".

Toda mudança gera algum desconforto, entretanto, uma vez que estas se tornem recorrentes, é necessário que o enfermeiro tenha 
habilidade para lidar com este tipo de situação. Mudanças programadas entre colaboradores, nos setores de maior incidência de ausência, podem minimizar o impacto das diferentes rotinas associadas a ações efetivas de educação permanente.

Outros aspectos pontuados negativamente pelos colaboradores foram a falta de objetividade na proposta de remanejamento e as consequências geradas pelo tempo dispendido entre detectar a necessidade do remanejamento e a efetivação do mesmo. Os entrevistados acreditam que esta conjuntura prejudica a assistência ao paciente.

TE 10: "[...] os pontos negativos giram em torno desse tempo que é gasto entre a definição de ser remanejado ou não".

TE 17: “[...] o atraso na medicação, atraso na orientação para o paciente [...] Aí, você chega lá nove ou dez horas da manhã, o paciente já tomou café e fez outras coisas".

TE 27: "[...] quando ele já chega e pega aquela escala e já começou o trabalho dele e depois tem que ser remanejado, tem que recomeçar tudo de novo[...]".

É importante percebermos que, quando os colaboradores estão insatisfeitos ou sobrecarregados em seu ambiente de trabalho, sentem-se desvalorizados e desmotivados ${ }^{(19)}$. Este contexto impacta diretamente na eficiência do processo de trabalho, uma vez que colaboradores satisfeitos, em suas atividades, tendem a faltar menos ao trabalho e a desenvolver suas funções com mais interesse ${ }^{(17)}$.

Há uma tendência de que o ambiente de trabalho se torne desumano em instituições onde, frequentemente, existem situações adversas $^{(17)}$. Associado a isto, ainda existem processos rotinizados pouco resolutivos e, muitas vezes, com o uso de formas desrespeitosas nas relações profissionais, agravando a situação e aumentando a possiblidade de riscos e vulnerabilidade. Neste sentido, é preciso refletir sobre este contexto, comum nas instituições hospitalares, e o manejo do processo de trabalho.

A reflexão pode favorecer a prevenção de problemas relacionados à diminuição $d a$ qualidade do serviço de enfermagem e ao aumento dos riscos ocupacionais.

\section{CONSIDERAÇÕES FINAIS}

Historicamente, na enfermagem, as reflexões sobre temas que permeiam as relações organizacionais tendem a ser tratadas de forma pouco aprofundada, haja vista o baixo número de publicações que tratam sobre a temática proposta, o que pode ser pontuado como uma limitação do estudo.

Esta investigação permitiu verificar que as percepções dos técnicos em enfermagem sobre o remanejamento relacionam-se com suas experiências profissionais e que esta prática traz grande impacto no processo de trabalho. Neste sentido, o remanejamento é percebido, pelos sujeitos desta pesquisa, como desconfortável, apesar de haver a compreensão da necessidade de tal ajuste.

Assim, faz-se necessária maior aproximação e discussão entre a equipe e gestão institucional, a fim de que as sequelas decorrentes do remanejamento sejam minimizadas, e que os profissionais técnicos em enfermagem possam ter espaço para a participação mais ativa nas tomadas de decisões. Estratégias como mapear os setores onde a incidência do remanejamento é maior, monitorar os funcionários que recorrem às faltas por diferentes motivos, construir indicadores consistentes, promover a melhora da comunicação interna associada a um processo de educação permanente efetivo tendem a gerar bons resultados.

\section{REFERÊNCIAS}

1 - Melo CMM, Florentino TC, Mascarenhas NB, Macedo K S, Silva MC, Mascarenhas SN. Autonomia profissional da enfermeira: algumas reflexões. Esc Anna Nery. 2016;20(4):e20160085. https://doi.org/10.5935/1414-8145.20160085

2 - Faria CC, Santos MCM, Luz NC, Pereira LF, Lima RS, Haddad JGV. How leader nurse communicates in a hospital: an analysis of discursive practices. Rev Pesq Cuid Fundam. 2017 jan/mar;9(1):152-8. https://doi.org/10.9789/2175-

\subsection{7.v9i1.152-158}

3 - Sancinetti TR, Soares AVN, Lima AFC, Santos NC, Melleiro MM, Fuguli $T$ et al. Taxa de absenteísmo da equipe de enfermagem como indicador de gestão de pessoas. Rev Esc Enferm USP.

2011;45(4):1007-12.

https://doi.org/10.1590/S0080-

62342011000400031

4 - Sales MLF, Okuno MFP, Campanharo CRV, Lopes MCBT, Belasco AGS, Batista REA. Prática profissional do enfermeiro em unidades críticas: avaliação das características do ambiente de trabalho. Rev Latinoam Enfermagem. 
2017;25:e2854. https://doi.org/10.1590/15188345.1424.2854

5 - Quadros DV, Magalhães AMM, Mantovani VM, Rosa DS, Echer IC. Análise de indicadores gerenciais e assistenciais após adequação de pessoal de enfermagem. Rev Bras Enferm. 2016;69(4):684-90.

http://dx.doi.org/10.1590/0034-

\subsection{0i}

6 - Trindade LL, Grisa CC, Ostrovski VP, Adamy EK, Ferraz L, Amestoy SC, Bordignon M. Absentismo en el equipo de enfermería en el ambiente hospitalario. Enferm Glob. 2014 [citado em 9 ago 2017];13(36):138-46. Disponível em: http://scielo.isciii.es/scielo.php?script=sci arttext \&pid=S1695-61412014000400008

7 - Schneider D, Winter V, Schreyögg J. Job demands, job resources, and behavior in times of sickness: an analysis across German nursing homes. Health Care Manage Rev. 2017 Mar 3. https://doi.org/10.1097/HMR.000000000000015 $\underline{7}$

8 - Kankaanranta T, Rissanen P. Nurses' intentions to leave nursing in Finland. Eur $\mathrm{J}$ Health Econ. 2014;9(4):333-42. https://doi.org/10.1007/s10198-007-0080-3

9 - Correia T. Interpretação e validação científica em pesquisa qualitativa. Interface (Botucatu). 2013;17(45):263-74.

https://doi.org/10.1590/S1414-

\section{2}

10 - Minayo MCS, Deslandes SF, Gomes R. Pesquisa Social: teoria, método e criatividade. Rio de Janeiro: Vozes; 2016.

11 - Bardin L. Análise de conteúdo. Lisboa, Portugal: Edições 70; 2015.

12 - Guerra ST, Prochnow AG, Trevizan MA, Guido LA. O conflito no exercício gerencial do enfermeiro no âmbito hospitalar. Rev Latinoam Enferm. 2011 [citado em 9 ago 2017];19(2):1-8. Disponível em: http://www.scielo.br/pdf/rlae/v19n2/pt 19.pdf

13 - Conselho Federal de Enfermagem. Resolução COFEN n. 543, de 18 de abril de 2017 [citado 3 nov 2016]. Atualiza e estabelece parâmetros para o Dimensionamento do Quadro de Profissionais de Enfermagem nos serviços/locais em que são realizadas atividades de enfermagem. Disponível em: http://www.cofen.gov.br/resolucao-cofen$\underline{543201751440 . \mathrm{html}}$

14 - Abreu RMD, Gonçalves RMDA, Simões ALA. Motivos atribuídos por profissionais de uma Unidade de Terapia Intensiva para ausência ao trabalho. Rev Bras Enferm. 2014;67(3):386-93. https://doi.org/10.5935/0034-7167.20140051

15 - Magalhães AMM, Dall'Agnol CM, Marck PB. Nursing workload and patient safety - a mixed method study with an ecological restorative approach. Rev Latinoam Enferm. 2013;21(no spe):146-54. https://doi.org/10.1590/S0104$\underline{11692013000700019}$

16 - Duclay E, Hardouin JB, Sébille V, Anthoine E, Moret L. Exploring the impact of staff absenteeism on patient satisfaction using routine databases in a university hospital. J Nurs Manag. 2015;23(7):833-41.

https://doi.org/10.1111/jonm.12219

17 - Fontana RT. Humanização no processo de trabalho em enfermagem: uma reflexão. Rev Rene. 2010 [citado em 10 fev 2017];11(1): 200-7. Disponível em: http://www.revistarene.ufc.br/vol11n1 html sit e/a21v11n1.htm

18 - Secco IAO et al. Cargas psíquicas de trabalho e desgaste dos trabalhadores de enfermagem de hospital de ensino do Paraná, Brasil. SMAD, Rev Eletr Saúde Mental Álcool Drogas. 2010 [citado em 10 fev 2017];;6(1):1-17. Disponível em: http://www.redalyc.org/pdf/803/80313414016.pdf 19 - Rodrigues AMS et al. O impacto das condições e jornada de trabalho na saúde dos trabalhadores de enfermagem. Rev Pesq Cuid Fundam. 2012 [citado em 9 ago 2017];4(4):2867-73. Disponível em:

http://www.seer.unirio.br/index.php/cuidadofun damental/article/viewFile/1398/pdf 627

Nota: Este estudo é produto de um projeto de pesquisa intitulado "Percepção dos técnicos de enfermagem sobre a necessidade de remanejamento entre setores em hospital de médio porte" que deu origem a um Trabalho de Conclusão de Curso no Centro Universitário Belo Horizonte com financiamento próprio.

Recebido em: $14 / 02 / 2017$

Aprovado em: 06/11/2017

Endereço de correspondência:

Humberto Ferreira de Oliveira Quites

UFSJ/Campus Centro Oeste - CCO

Rua Sebastião Gonçalves Coelho, no 400, sala 306.2, Bloco D Bairro Chanadour

CEP: 35.501-296 - Divinópolis/MG - Brasil

E-mail: hquites@ufsj.edu.br 